\title{
Research on Effect and Mechanism of Xuefu Zhuyu Decoction on CHD Based on Meta-Analysis and Network Pharmacology
}

\author{
Fuguang Kui, ${ }^{1}$ Wenwen Gu, ${ }^{1}$ Fan Gao, ${ }^{1}$ Yuji Niu, ${ }^{1}$ Wenwen Li, ${ }^{1}$ Yaru Zhang, ${ }^{1}$ Lijuan Guo, \\ Junru Wang, ${ }^{1}$ Zhenzhen Guo, ${ }^{1}$ Shihong Cen $\mathbb{D D}^{2},{ }^{2}$ and Gangjun Du $\mathbb{I D}^{1,2}$ \\ ${ }^{1}$ Institute of Pharmacy, Pharmaceutical College of Henan University, Jinming District, Kaifeng, Henan 475004, China \\ ${ }^{2}$ School of Pharmacy and Chemical Engineering, Zhengzhou University of Industry Technology, Xinzheng, Henan 451150, China
}

Correspondence should be addressed to Shihong Cen; 10200053@vip.henu.edu.cn and Gangjun Du; 10200029@vip.henu.edu.cn

Received 28 July 2020; Revised 4 November 2020; Accepted 8 December 2020; Published 13 February 2021

Academic Editor: Yong Wang

Copyright (c) 2021 Fuguang Kui et al. This is an open access article distributed under the Creative Commons Attribution License, which permits unrestricted use, distribution, and reproduction in any medium, provided the original work is properly cited.

\begin{abstract}
Xuefu Zhuyu Decoction (XFZY) is an ancient compound widely used in the treatment of coronary heart disease. However, its efficacy evaluation is not complete and its mechanism of action is not clear enough. In an attempt to address these problems, the efficacy was evaluated by meta-analysis and the mechanism was elucidated by the network pharmacology method. We systematically searched relevant studies in PubMed, Chinese National Knowledge Infrastructure Database (CNKI), Cochrane Library, Wanfang Data, and other databases from 2007 to 2019. The association between XFZY treatment and CHD was estimated by risk ratio (RR) and corresponding $95 \%$ confidence intervals (95\% CIs). The compounds and the potential protein targets of XFZY were obtained from TCMSP, and active compounds were selected according to their oral bioavailability and drug similarity. The potential genes of coronary heart disease were obtained from TTD, OMIM, and GeneCards. The potential pathways related to genes were determined by GO and KEGG pathway enrichment analyses. The compound-target and compound-target-pathway networks were constructed. Molecular docking validates the component and the target. A total of 21 studies including 1844 patients were enrolled in the present meta-analysis, indicating that XFZY has a greater beneficial on total effect (fixed effect $\mathrm{RR}=1.30 ; 95 \% \mathrm{Cl}: 1.24-1.36 ; P=0.82 ; I^{2}=0.0 \%$ ) and electrocardiogram efficacy (fixed effect $\mathrm{RR}=1.40 ; 95 \% \mathrm{Cl}: 1.26-1.56$; $\left.P=0.96 ; I^{2}=0.0 \%\right)$ compared with the control group. A total of 1342 components in XFZY were obtained, among which, 241 were chosen as bioactive components. GO and KEGG analyses got top 10 significantly enriched terms and 10 enriched pathways. The C-T network included 192 compounds and 3085 targets, whereas the C-T-P network included 10 compounds, 109 targets, and 5 pathways. There was a good binding activity between the components and the targets. XFZY has the curative effect on coronary heart disease, and its mechanism is related to 10 compounds, 10 core targets, and 5 pathways.
\end{abstract}

\section{Introduction}

Coronary heart disease (CHD) is a leading cause of death and disability worldwide [1]. CHD is caused by narrowing or obstruction of blood vessels due to coronary atherosclerosis, myocardial ischemia, hypoxia, and necrosis. CHD has intervened effectively with the advances in cardiovascular medicine over the past decades. Despite great improvements in cardiovascular medicine, these developments now still generate to post-treat sequelae. Therefore, finding a more effective therapy method is an urgent need.
Traditional Chinese medicine (TCM), embracing centuries of knowledge and wisdom, is a medical practice [2] and has played a significant therapeutic role in various diseases [3]. Xuefu Zhuyu Decoction (XFZYD), from "Yiling Gaicuo," is Chinese herbal formulas commonly used to treat hypertension and cardiovascular diseases in traditional Chinese medicine [4]. It consists of eleven herbs, namely, Paeonia lactiflora Pall (Chi Shao in Chinese); Ligusticum chuanxiong Hort (Chuan Xiong in Chinese); Bupleurum chinensie DC (Chai Hu in Chinese); Carthamus tinctorius L. (Hong Hua in Chinese); Angelica sinensis (Oliv) Diels 
(Dang Gui in Chinese); Prunus persica (L.) Batsch (Tao Ren in Chinese); Achyranthes bidentata BI (Niu Xi in Chinese); Glycyrrhiza uralensis Fisch (Gan Cao in Chinese); Platycodon grandifloras (Jacq.) A. DC (Jie Geng in Chinese); Citrus aurantium L. (Zhi Ke in Chinese); Rehmannia glutinosa Libosch (Sheng Di Huang in Chinese). According to clinical view of TCM, Qi is the commander of blood. Qi stagnation causes blood stasis, which leads to CHD [5]. Studies have shown that XFZY is regulating Qi and promoting blood circulation [6]. Clinical studies have shown that XFZY is effectively ameliorative to the clinical symptoms of CHD without side effect [7]. The therapeutic effects of XFZY on atherosclerosis and hyperlipidemia were validated [8]. XFZY will improve the phlegm and blood stasis pattern in CHD [9]. XFZY is very effective in the treatment myocardial fibrosis, atherosclerosis, hypertension, unstable angina pectoris, and myocardial ischemia-reperfusion injury [10]. However, these studies are not supported by a large amount of clinical data, or incomplete. Therefore, this paper will use meta-analysis to evaluate the efficacy of XFZY on CHD through the study of a large number of clinical data. Currently, network pharmacology has shown that for complex diseases, Chinese medicine formulas have the advantages of multitarget interventions and minimal side effect [11]. Therefore, this study will also perform network pharmacology to elucidate the mechanism of XFZY on CHD.

\section{Methods}

The reporting of this study was guided by the Preferred Reporting Items for Systematic Reviews and Meta-Analyses checklist. The registration application for the systematic review protocol has been submitted at PROSPERO, but the registration number has not been obtained.

2.1. Literature Search. We searched the main literature database at home and abroad, namely, PubMed, PubMed Pro, Embase, Chinese Scientific Journal Database (VIP), SinoMed, Cochrane Library, CNKI, and Wanfang Data, from 2007 to 2019, identifying available studies to be included in the meta-analysis. The keywords that were used are as follows: Xuefu Zhuyu Tang, Xuefu Zhuyu Decoction, XFZY, XFZYD, coronary heart disease, and CHD. Besides, we also screened all other possible reference lists from the studies selected to identify further relevant studies and reviews (see Supplementary File 1 for further information).

2.2. Criteria for Literature Inclusion. The inclusion criteria were as follows:

(1) Included studies were required to have enrolled patients with a clear diagnosis of coronary heart disease; no restrictions on race, age, or sex were imposed

(2) Randomized, clinical trial
(3) Control group for routine treatment and experimental group for XFZY on the basis of the control group

(4) Basis of disease diagnosis is Nomenclature and Diagnostic Criteria for Ischemic Heart Disease and Guiding Principles for Clinical Research of New Chinese Medicines (trial)

(5) The course of treatment is four weeks or more

(6) It conforms to the ethical and moral treatment standard

(7) The patient has no other mental illness or serious primary disease

(8) The patient is not lactation, pregnancy, or advanced stage of disease

(9) One or more outcome indicators of the following must be involved: (1) total therapeutic effect; (2) ECG; (3) adverse reaction; (4) angina pectoris attack frequency and duration; (5) the total effect of TCM syndromes; (6) serum lipids index; (7) blood stream change; (8) the effect of electroacupuncturing acupoint; (9) vessel endothelial function and factors of vessel endothelium; (10) LVEF and PAF; (11) average hospital stay; (12) disappeared time of angina; (13) effects of decreased dose; (14) symptom improvement; (15) the total angina pectoris efficacy.

Therefore, exclusion criteria included the following:

(1) Republished studies

(2) Nonrandomized trials

(3) Experiments on animals

(4) Review

(5) There are no clear diagnostic criteria or not meet inclusion criteria

(6) Lack of required data for meta-analysis

(7) Treatment duration is less than four weeks

(8) There is no complete evaluation of efficacy

2.3. Data Extraction. Two authors searched the literature according to the established strategy, then extracted data from the included studies, and compared the results independently. Discrepancy was resolved by the third author's adjudication to avoid bias. Extracted data included author, year of publication, title of the study, diagnostic criteria ,sample size, course of treatment, intervention and control measures, evaluation standard, random scheme generation, allocation hiding, blind method, incomplete result data, selective reporting, and other biases.

2.4. Quality Assessment. The Cochrane Collaboration's Bias Risk Assessment Tool was used to assess the quality of the literature. The main assessment areas are as follows: allocation concealment, random sequence generation, incomplete outcome data, blinding method for patients/ researchers and outcomes assessors, selective reporting, and 
other sources of bias. The results were judged as "low risk," "high risk," and "unclear."

2.5. Statistical Analysis. Statistical analyses were conducted using RevMan5.3 (Review Manager 5.3). Between studies, heterogeneity of each study was assessed using $I^{2}$ tests. If high heterogeneity $\left(I^{2}>50 \%\right)$ was observed, random effect models were applied; otherwise, fixed effect models were used. Pooled RR with corresponding 95\% CIs was used to evaluate the effect of XFZY on CHD. We used a funnel plot to determine potential publication bias.

2.6. Active Ingredients. Compounds of ten ingredients in XFZY were gathered from TCM Systems Pharmacology Database (TCMSP, a unique systems pharmacology platform designed for herbal medicines). Compounds of Sheng Di Huang were obtained from Chemistry Database (http:// www.organchem.csdb.cn/). The ADME (absorption, distribution, metabolism, and excretion) was used to filtrating active compounds. The active components were obtained by screening for both oral bioavailability (OB) value $\geq 30 \%$ and drug similarity (DL) value $\geq 0.18$.

2.7. Potential Target Identification. The targets of the active ingredients in XFZY were obtained from the TCMSP database and Chemistry Database (http://www.organchem. $\operatorname{csdb.cn/).~}$

2.8. CHD-Related Gene Targets. Retrieving the Therapeutic Target Database (TTD, http://db.idrblab.net/ttd/), Online Mendelian Inheritance in Man (OMIM https://www.omim. org/), and GeneCards (https://www.genecards.org/) about CHD, CHD-related gene targets were collected.

2.9. Gene Ontology (GO) and KEGG Pathway Enrichment. To seek out the biological characteristics of bioactive targets of XFZY on CHD in detail, the GO and KEGG pathway enrichment analyses of bioactive targets were conducted via DAVID 6.8 (https://david.ncifcrf.gov/). The top 10 significantly enriched terms and 10 significantly enriched pathways of XFZY on CHD were visualization.

2.10. Network Construction and Analysis. To further characterize the mechanism of XFZY on CHD, the C-T and C-T$\mathrm{P}$ networks were constructed via Cytoscape 3.2.1. In these networks, compounds, targets, and pathways were expressed as nodes, whereas the C-T and C-T-P interactions were expressed as edges.

2.11. Molecular Docking. The bioactive component and the target with the highest degree value were selected for molecular docking. Mol2 format of molecular structure of bioactive components was obtained from the TCMSP database, and PDB format of 3D molecular structure of corresponding target genes was obtained from the RCSB PDB database (https://www.rcsb.org/). LeDock software was used to dock the bioactive components with the target to obtain the docking affinity. SwissDock (http://www.swissdock.ch/) and Chimera 1.14 software were used to perform molecular docking visualization analysis of compounds with high docking affinity and stable conformation with target proteins.

\section{Results}

3.1. Search Results and Study Quality. Figure 1 schematically shows the selection procedure for eligible articles. 238 references were obtained by searching all databases, among them, 50 papers from SinoMed, 110 papers from CNKI, and 78 papers from Wanfang Data. Removed duplicates, a total of 128 papers were identified in the initial search. However, 107 articles were excluded due to irrelevant content or not up to inclusion criteria after reading the titles and abstracts (shown in Supplementary File 4). After evaluation, 21 papers qualified for detailed evaluation. A total of 1844 patients from 21 included studies, 919 control groups and 925 treatment groups, were included in this meta-analysis. The detail data extracted from these included articles are listed in Table 1. Quality assessment of 21 available studies was performed by using the Risk Assessment Tool of the Cochrane Library. The risk of inclusion literature is shown in Figure 2.

3.2. Heterogeneity Detection and Pooled Analysis. We included 21 RCTs with a total of 1844 participants. All the 21 studies, including a total of 1844 patients, reported total effect of XFZY on CHD. We used a fixed effect model to analyze results because heterogeneity was low $\left(I^{2}<50 \%\right)$. Meta-analysis showed that patients with XFZY had more effective than control group (Figure 3) (fixed effect $\left.\mathrm{RR}=1.30 ; 95 \% \mathrm{Cl}: 1.24-1.36 ; P=0.82 ; I^{2}=0.0 \%\right) .8$ studies, including a total of 674 patients, reported electrocardiogram efficacy of XFZY on CHD. We used a fixed effect model to analyze results. Meta-analysis showed that, compared with the control group, the experimental group with XFZY was more effective than the control group (Figure 4) (fixed effect $\left.\mathrm{RR}=1.40 ; 95 \% \mathrm{Cl}: 1.26-1.56 ; P=0.96 ; I^{2}=0.0 \%\right)$.

3.3. Publication Bias. Funnel plots were made to evaluate publication bias. The result indicated that no clear bias in the total effect (Figure 5(a)) and electrocardiogram efficacy (Figure 5(b)).

3.4. Potential Active Moieties of XFZY. A total of 1342 chemical moieties obtained from the XFZY formula were collected from TCMSP. By ADME filtering, only 222 compounds met the principle of both oral bioavailability $(\mathrm{OB})$ value $\geq 30 \%$ and drug similarity (DL) value $\geq 0.18$. The number of potential active compounds from $\mathrm{CS}, \mathrm{CX}, \mathrm{CH}$, HH, DG, TR, NX, GC, JG, and ZK was 27, 7, 17, 22, 2, 23, 20, 92 , and 7,5 , respectively. 19 chemical moieties of SD were collected from the Chemistry Database (shown in 


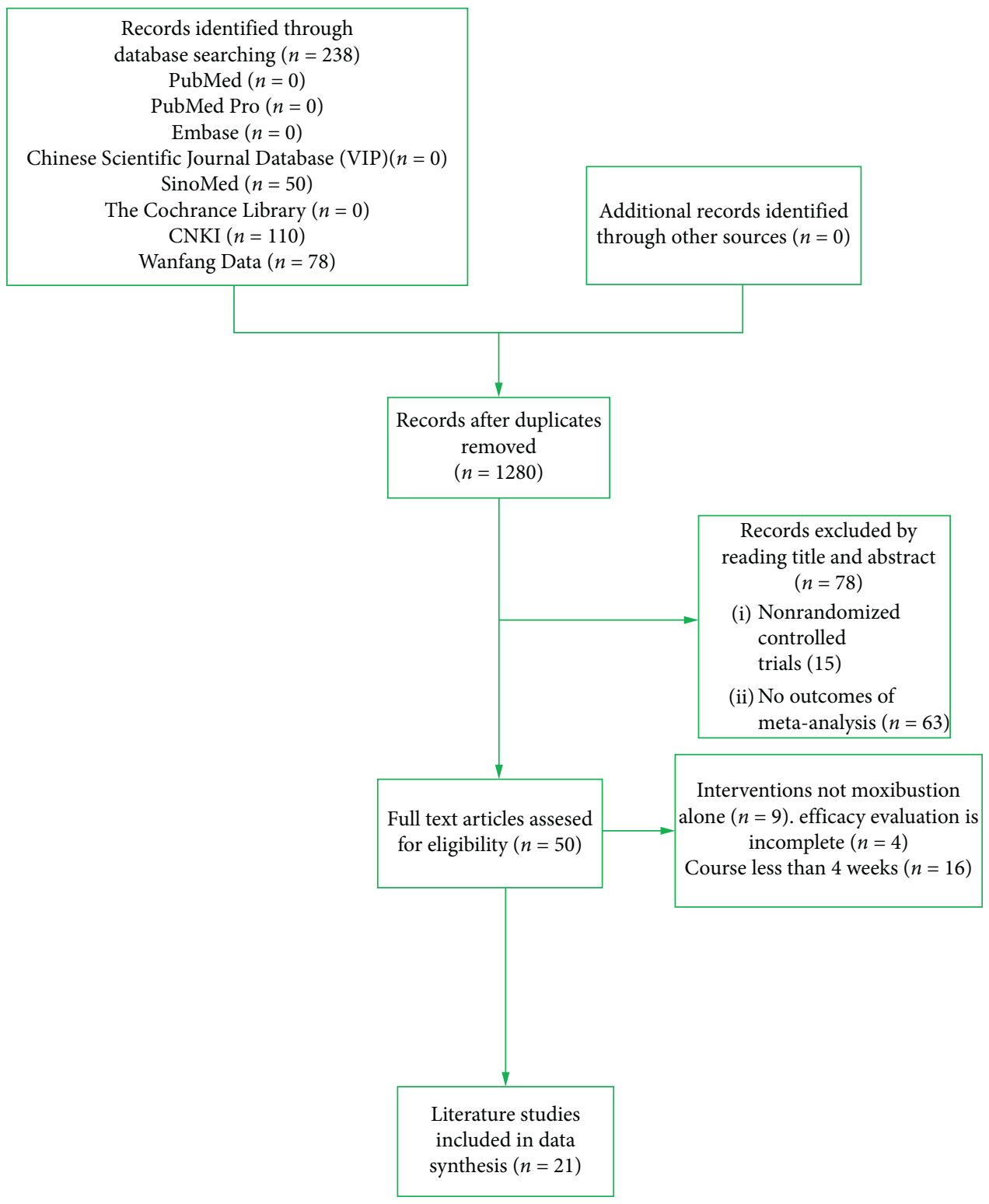

FIgURE 1: The flowchart of the study selection and exclusion criteria.

TABLe 1: The characteristics of all included studies.

\begin{tabular}{lccccccc}
\hline Author and reference & Year & Total sample & Control/treatment & $\begin{array}{c}\text { Period of } \\
\text { treatment (day) }\end{array}$ & Drug & C & T \\
\hline Li Jiayun [12] & 2019 & 108 & $54 / 54$ & 60 & $(1),(4),(6)$ & $(11)$ & $1,3,9$ \\
Lu Jiansheng [13] & 2018 & 64 & $32 / 32$ & 60 & $(1),(3),(6)$ & $(11)$ & $1,3,7,8,11$ \\
Jiao Lixing [14] & 2015 & 78 & $39 / 39$ & 30 & $(1),(10)$ & $(11)$ & $1,3,8,12$ \\
Zhao Dongkai [15] & 2015 & 100 & $50 / 50$ & 30 & $(14)$ & $(11)$ & $1,2,4$ \\
Huang Wenhua [16] & 2018 & 71 & $35 / 36$ & 30 & $(1),(2)$ & $(11)$ & 1,4 \\
Li Tingai [17] & 2019 & 110 & $55 / 55$ & 30 & $(1),(2),(3)(3)$ & $(11)$ & 1,5 \\
Fang Yuxiang [18] & 2009 & 117 & $57 / 60$ & 28 & $(1),(2)(2)$ & $(11)$ & $1,7,8,13$ \\
Ying Ping [19] & 2018 & 100 & $50 / 50$ & 60 & $(1),(3),(4)$ & $(11)$ & $1,6,7$ \\
Wang Baoxin [20] & 2010 & 58 & $29 / 29$ & 30 & $(1),(8)$ & $(11)$ & 1,4 \\
Fang Jianjia [21] & 2008 & 60 & $30 / 30$ & 28 & $(2),(3)$ & $(11)$ & $1,2,14$ \\
Lin Zhijuan [22] & 2015 & 70 & $30 / 40$ & 30 & $(1),(2),(3),(9),(13)$ & $(11)$ & $2,6,15$ \\
Song Yuxin [23] & 2013 & 82 & $40 / 42$ & 28 & $(1),(6),(7),(9)$ & $(11)$ & $1,2,5,7$ \\
Lv Xiqi [24] & 2019 & 100 & $50 / 50$ & 60 & $(1),(3),(5)$ & $(11)$ & 1,7 \\
\hline
\end{tabular}


TABLE 1: Continued.

\begin{tabular}{lccccccc}
\hline Author and reference & Year & Total sample & Control/treatment & $\begin{array}{c}\text { Period of } \\
\text { treatment (day) }\end{array}$ & Drug & Evaluation standard \\
\hline Li Guangcheng [25] & 2009 & 80 & $40 / 40$ & 30 & $(1),(2)$ & $(11)$ & $1,2,5$ \\
Yang Suqing [26] & 2015 & 96 & $48 / 48$ & 28 & $(1),(2),(3)$ & $(11)$ & $1,3,5$ \\
Zhang Guangyu [27] & 2018 & 92 & $46 / 46$ & 28 & $(1),(3),(8)$ & $(11)$ & $1,3,4$ \\
Liang Lifeng [28] & 2015 & 100 & $50 / 50$ & 28 & $(6),(7),(9)$ & $(11)$ & $1,2,7$ \\
Cai Xiaoming [29] & 2017 & 96 & $48 / 48$ & 28 & $(6),(7),(9)$ & $(11)$ & $(1),(7)$ \\
Zhou Fei [30] & 2019 & 80 & $40 / 40$ & 28 & $(2),(7),(9)$ & $(11)$ & $1,2,6$ \\
Zhang Mingli [31] & 2010 & 80 & $40 / 40$ & 28 & $(3),(4),(10)$ & $(11)$ & $1,2,7$ \\
Cheng Lisheng [32] & 2015 & 92 & $46 / 46$ & 28 & 4,7 \\
\hline
\end{tabular}

Abbreviations: (1) aspirin; (2) nitroglycerin; (3) isosorbide dinitrate/isosorbide mononitrate; (4) metoprolol; (5) nifedipine; (6) nitrates; (7) $\beta$-blocker; (8) Betocloc; (9) calcium antagonist; (10) captopril; (11) Xuefu Zhuyu Decoction based on the control group; (12) lipid-lowering agents and pain relief drugs; (13) clopidogrel and atorvastatin; (14) Compound Salvia Tablets. 1 Total therapeutic effect; 2 ECG; 3 adverse reaction; 4 angina pectoris attack frequency and duration; 5 the total effective of TCM syndromes; 6 serum lipid index; 7 blood stream change; 8 the effect of electroacupuncturing acupoint; 9 vessel endothelial function and factors of vessel endothelium; 10 LVEF and PAF; 11 average hospital stay; 12 disappeared time of angina; 13 effects of decreased dose; 14 symptom improvement; 15 the total angina pectoris efficacy.

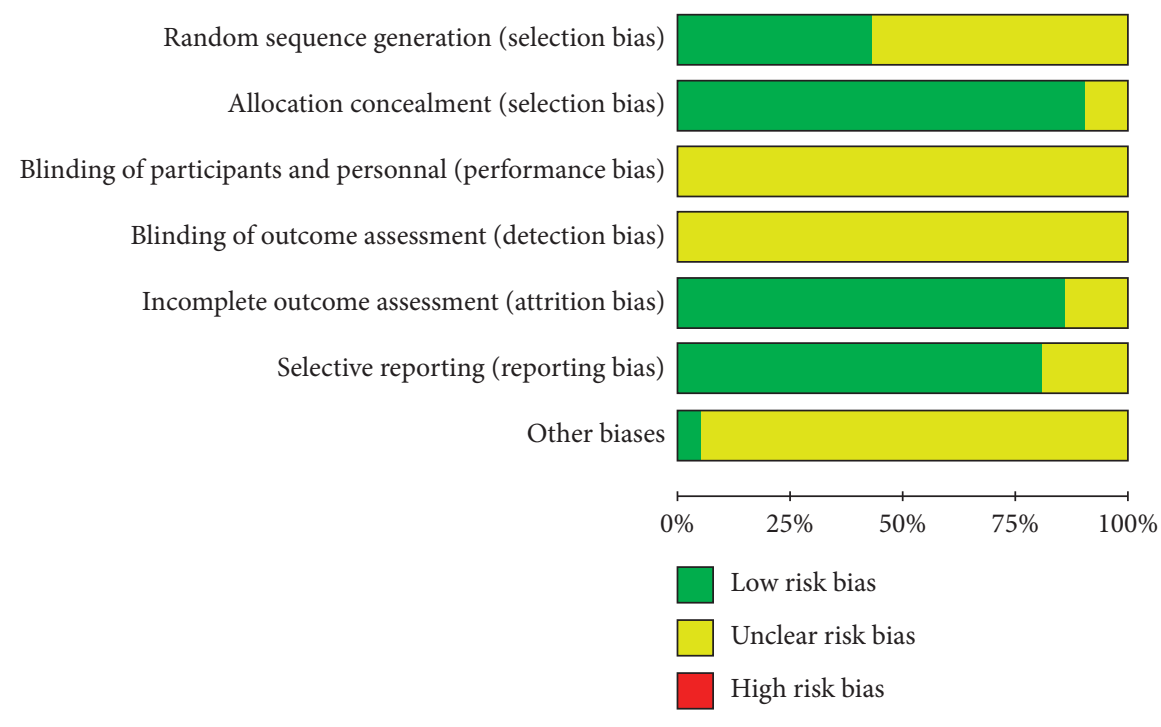

(a)

Figure 2: Continued. 


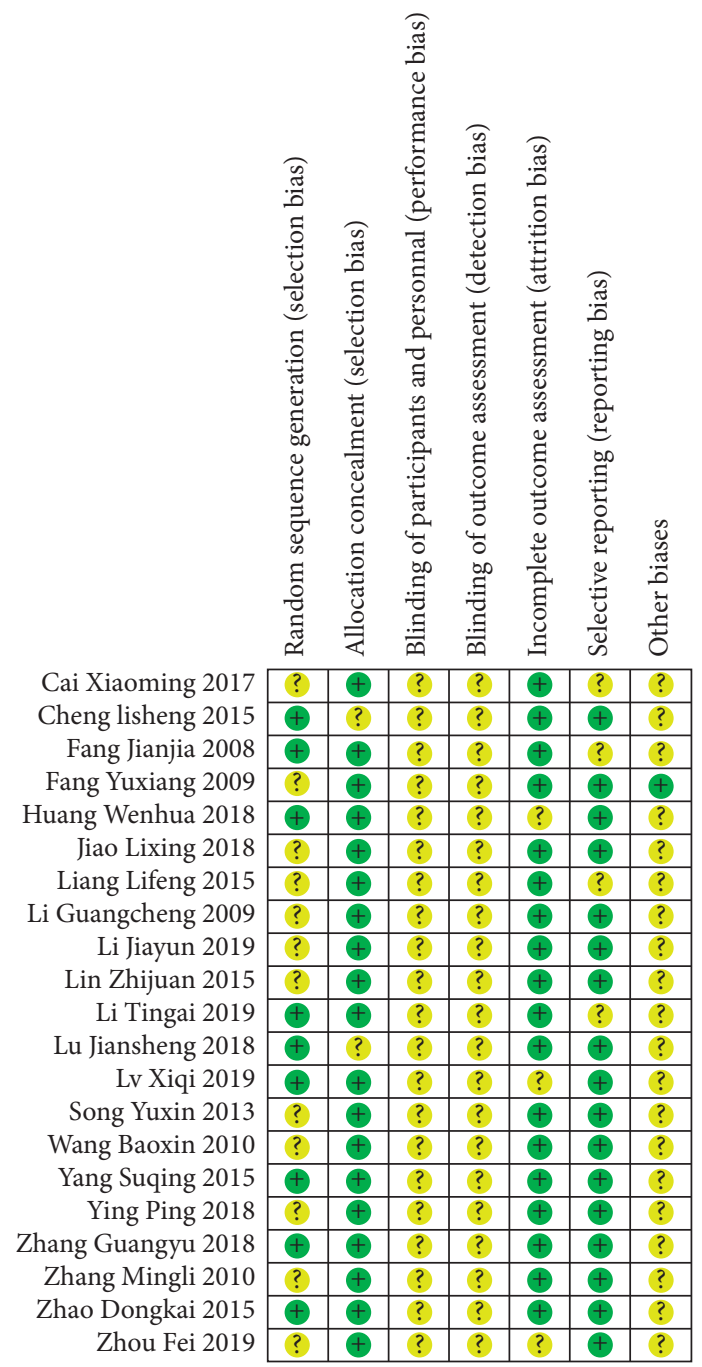

(b)

Figure 2: Risk bias graph of studies.

Supplementary File 2). The compounds were chosen as potential active moieties for further analyses.

3.5. Putative Targets for the Active Compounds of XFZY. Putative targets corresponding to 241 components were obtained from TCMSP and Chemistry Database. Removing duplicates, 163 protein targets were obtained for further analyses (shown in Supplementary File 3).

3.6. Targets of Coronary Heart Disease. In total, 6785 targets of CHD were obtained from TTD, OMIM, and GeneCards (shown in Supplementary File 5).

3.7. GO and Pathway Enrichment Analysis. DAVID 5.6 was used to perform the GO and KEGG pathway enrichment of XFZY for CHD. The top 10 significantly enriched terms are shown (Figure 6(a)), which exerted its therapeutic effects on CHD involving "response to drug," "positive regulation of nitric oxide biosynthetic process," "response to hypoxia," "positive regulation of cell proliferation," "positive regulation of cell migration," "response to estradiol," "positive regulation of transcription," "angiogenesis," and "aging biological process." The top 10 significantly enriched pathways of XFZY on CHD are shown (Figure 6(b)).

\subsection{Network Construction and Analysis}

3.8.1. Compound-Target Network (C-T Network). We performed bioactive target identification via WebGestalt (http://webgestalt.org/). There were 124 protein targets, which were the intersection of two parts, the targets of active compounds, and the targets of CHD, defined as bioactive targets for XFZY treating CHD. There were 192 corresponding compounds of bioactive targets. The compoundtarget network was constructed via Cytoscape3.2.1. The nodes represent compounds, targets, and edges which represent their interaction (Figure 7). The node color reflects 


\begin{tabular}{|c|c|c|c|c|c|c|c|c|c|c|c|}
\hline \multirow{2}{*}{$\begin{array}{l}\text { Study or subgroup } \\
\text { Cai Xiaoming } 2017\end{array}$} & \multicolumn{2}{|c|}{ Experimental } & \multicolumn{2}{|c|}{ Control } & $\begin{array}{l}\text { Weight } \\
(\%)\end{array}$ & $\begin{array}{l}\text { Risk ration } \\
\text { M-H, fixed, } 95 \% \text { CI }\end{array}$ & \multicolumn{5}{|c|}{$\begin{array}{c}\text { Risk ration } \\
\text { M-H, fixed, } 95 \% \text { CI }\end{array}$} \\
\hline & 45 & 48 & 38 & 48 & 5.8 & $1.18[1.01,1.39]$ & & & & & \\
\hline Cheng lisheng 2015 & 44 & 46 & 36 & 46 & 5.5 & $1.22[1.04,1.44]$ & & & & & \\
\hline Fang Jianjia 2008 & 25 & 30 & 18 & 30 & 2.7 & $1.39[1.00,1.94]$ & & & & & \\
\hline Fang Yuxiang 2009 & 54 & 60 & 31 & 57 & 4.8 & $1.65[1.29,2.13]$ & & & & & \\
\hline Huang Wenhua 2018 & 33 & 36 & 26 & 35 & 4.0 & $1.23[0.99,1.54]$ & & & & & \\
\hline Jiao Lixing 2018 & 36 & 39 & 28 & 39 & 4.3 & $1.29[1.04,1.60]$ & & & & & \\
\hline Li Guangcheng 2009 & 38 & 40 & 28 & 40 & 4.3 & $1.36[1.09,1.68]$ & & & & & \\
\hline Li Jiayun 2019 & 51 & 54 & 40 & 54 & 6.1 & $1.27[1.08,1.51]$ & & & & & \\
\hline Li Tingai 2019 & 52 & 55 & 44 & 55 & 6.7 & $1.18[1.08,1.51]$ & & & & & \\
\hline Liang Lifeng 2015 & 44 & 50 & 33 & 50 & 5.0 & $1.33[1.07,1.67]$ & & & & & \\
\hline Lin Zhijuan 2015 & 38 & 40 & 26 & 40 & 4.0 & $1.46[1.15,1.85]$ & & & & & \\
\hline Lu Jiansheng 2018 & 29 & 32 & 21 & 32 & 3.2 & $1.38[1.05,1.82]$ & & & & & \\
\hline Lv Xiqi 2019 & 48 & $\begin{array}{l}50 \\
42\end{array}$ & 39 & 50 & 5.9 & $1.23[1.05,1.44]$ & & & & & \\
\hline Song Yuxin 2013 & 37 & $\begin{array}{l}42 \\
29\end{array}$ & 27 & 40 & 4.2 & $1.31[1.02,1.66]$ & & & & & \\
\hline Wang Baoxin 2010 & 27 & $\begin{array}{l}29 \\
48\end{array}$ & 20 & 29 & 3.0 & $1.35[1.04,1.76]$ & & & & & \\
\hline Yang Suqing 2015 & 45 & 50 & 35 & 48 & 5.3 & $1.29[1.07,1.55]$ & & & & & \\
\hline Ying Ping 2018 & 48 & 46 & 36 & 50 & 5.5 & $1.33[1.11,1.60]$ & & & & & \\
\hline Zhang Guangyu 2018 & 44 & 40 & 38 & 46 & 5.8 & $1.16[1.00,1.34]$ & & & • & & \\
\hline Zhang Mingli 2010 & 33 & 40 & 25 & 40 & 3.8 & $1.32[1.00,1.75]$ & & & & & \\
\hline Zhao Dongkai 2015 & 46 & 50 & 33 & 50 & 5.0 & $1.39[1.12,1.73]$ & & & & & \\
\hline Zhou Fei 2019 & 38 & 40 & 33 & 40 & 5.0 & $1.15[0.98,1.35]$ & & & $\bullet$ & & \\
\hline Total (95\% CI) & & 925 & & 919 & 100.0 & $1.30[1.24,1.36]$ & & & & & \\
\hline Total events & 855 & & 655 & & & & & & & & \\
\hline \multirow{2}{*}{\multicolumn{7}{|c|}{$\begin{array}{l}\text { Heterogeneity: } \text { chi }^{2}=14.19, d f=20(P=0.82) ; I^{2}=0 \% \\
\text { Test for overall effect: } Z=11.40(P<0.00001)\end{array}$}} & 0.5 & 0.7 & 1 & 1.5 & $\begin{array}{l}1 \\
2\end{array}$ \\
\hline & & & & & & & Favour & perimental) & Favours ( & (control) & \\
\hline
\end{tabular}

Figure 3: Comparison of total effect between the experimental group and the control group for coronary heart disease.

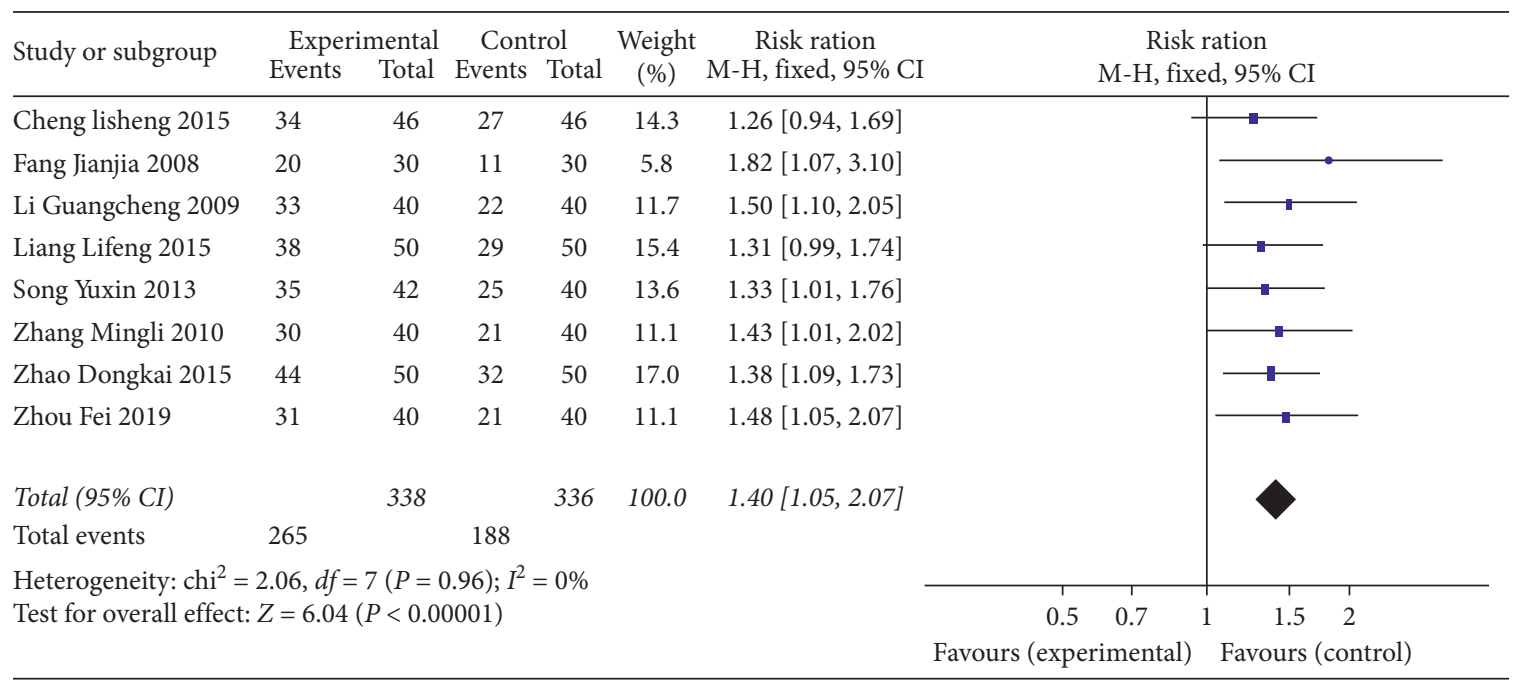

FiguRE 4: Comparison electrocardiogram efficacy between the experimental group and the control group for coronary heart disease.

the degree value. The edge thickness reflects the correlation between nodes. The result suggested that the top $10 \mathrm{com}-$ pounds are as follows: quercetin; 7-methoxy-2-methyl isoflavone; (2R)-7-hydroxy-2-(4-hydroxyphenyl)chroman-4one; beta-sitosterol; kaempferol; luteolin; medicarpin; formononetin; shinpterocarpin; 2-[(3R)-8,8-dimehyl-3,4dihydro-2H-pyrano[6,5-f]chromen-3-yl]-5-mehoxyphenol.
It means that these compounds may play a major role in the therapeutic effects. The top 10 targets are as follows: AR, ESR1, PTGS2, NOS2, PPARG, PIM1, DPP4, GSK3B, PRSS1, and ESR2. These targets may be the main targets of drug action. In addition, it can be seen that an effective component corresponds to multiple targets, and a target corresponds to multiple effective components. 


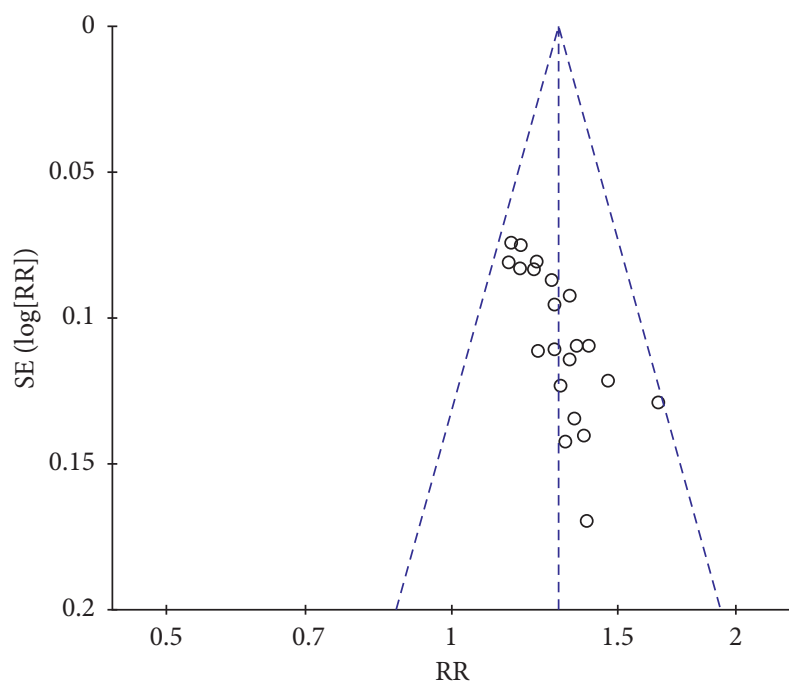

(a)

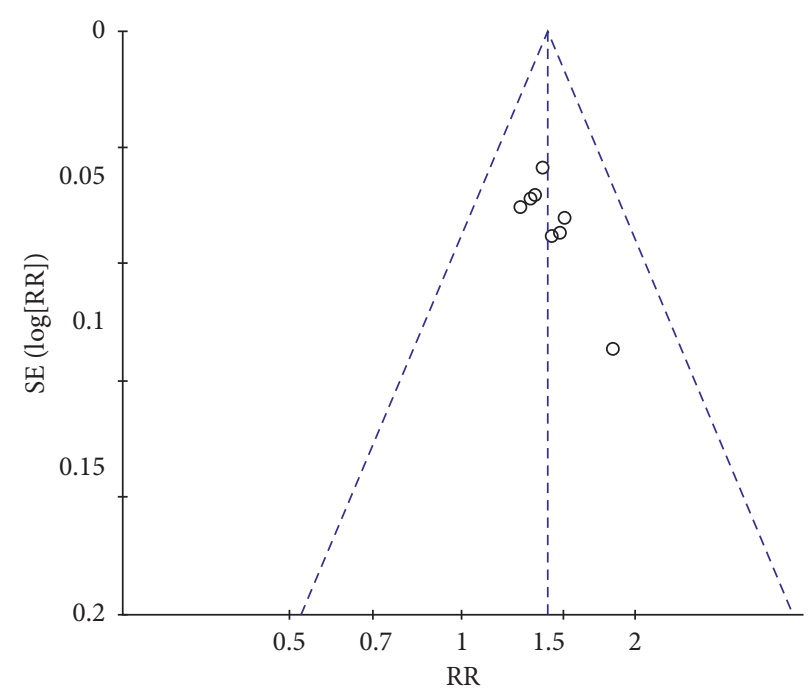

(b)

Figure 5: (a) The bias of the total effect in the treatment of coronary heart disease by Xuefu Zhuyu Decoction; (b) the bias of electrocardiogram efficacy in the treatment of coronary heart disease by Xuefu Zhuyu Decoction.

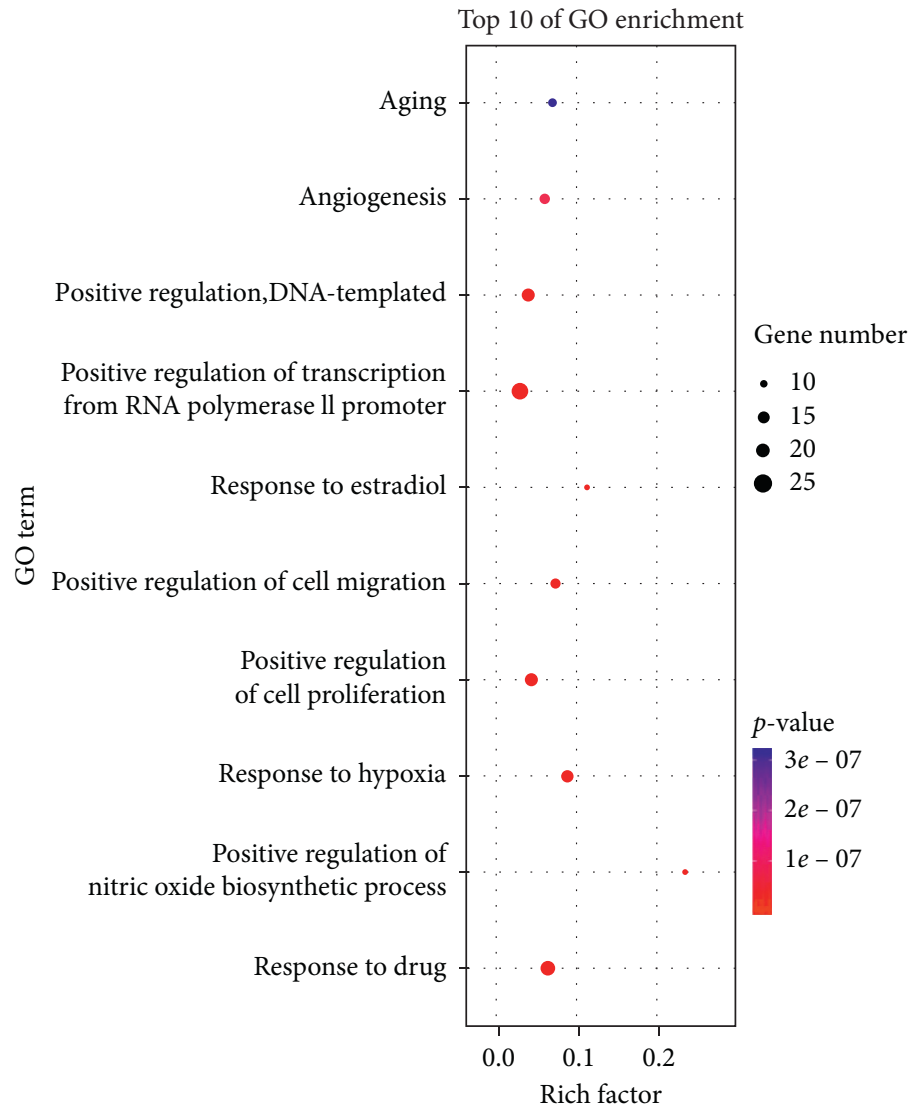

(a)

Figure 6: Continued. 


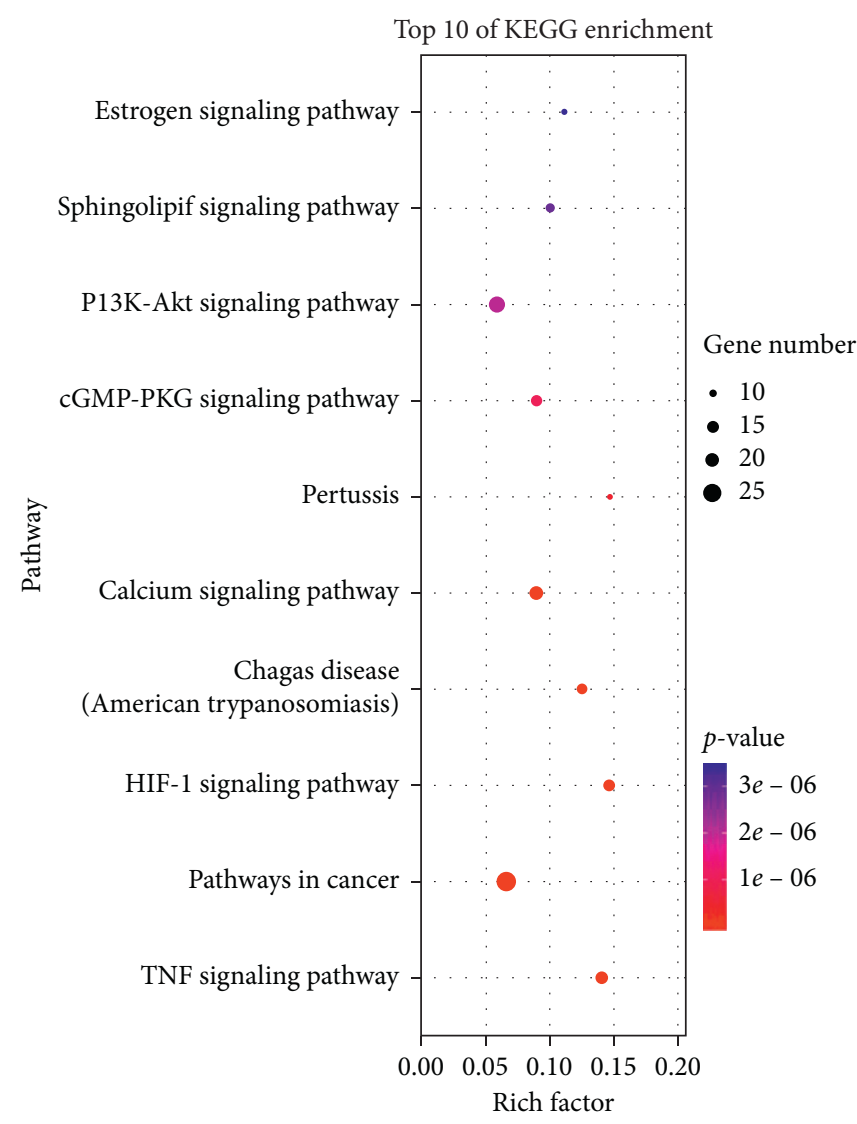

(b)

FIGURE 6: The 10 most significance of gene ontology (a) and pathway enrichment (b) analysis of therapy target genes of XFZY on CHD.

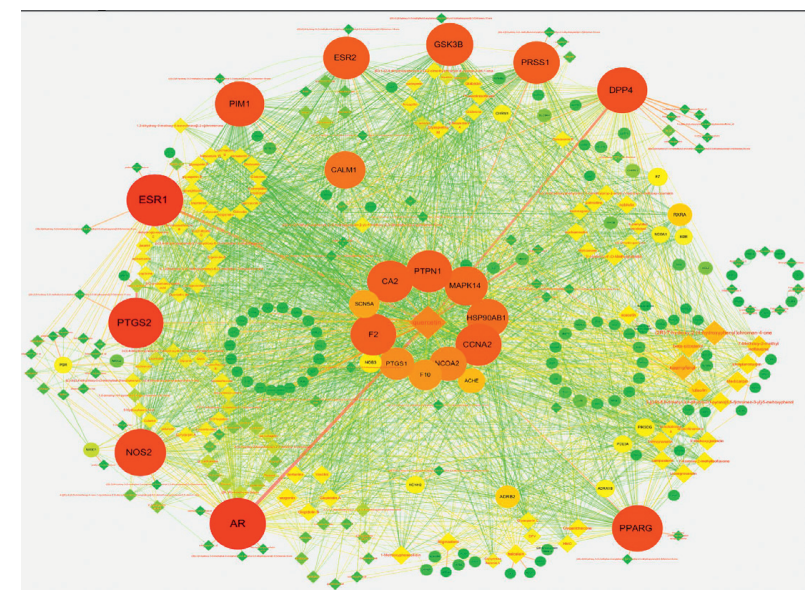

Figure 7: The compound-target network of XFZY on CHD. The circles represent potential protein targets, and the rhombus represents bioactive compounds. The edges represent the interactions between them, and nodes' sizes are proportional to their degree.

3.8.2. Compound-Target-Pathway Network (C-T-P Network). To further characterize the molecular mechanism by which XFZY on CHD, a compound-target-pathway network was performed based on all involved compounds, targets, and their corresponding significant signal pathways. Therefore, five signal pathways that may be related to $\mathrm{CHD}$ were selected from the top 10 KEGG genes in the treatment of CHD by XFZY, constructing the component-target-pathway network (Figure 8). The five signal pathways are follows: TNF signaling pathway, pathways in cancer, calcium signaling pathway, HIF-1 signaling pathway, and Chagas disease (American trypanosomiasis), respectively. 


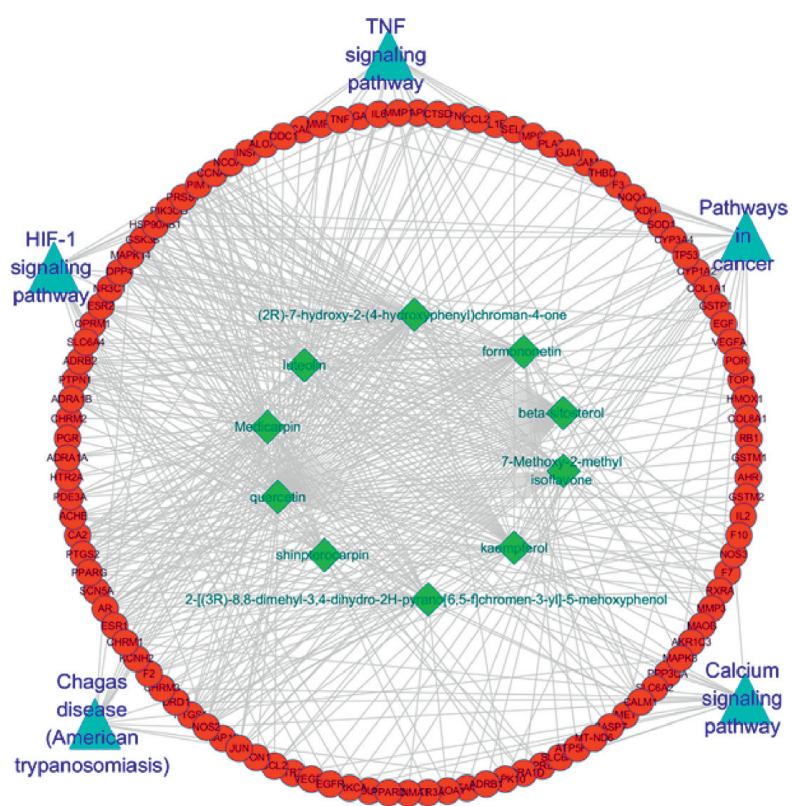

FIGURE 8: The compound-target-pathway network of XFZY on CHD. The read circles represent potential protein targets, the green rhombuses represent bioactive compounds, and the blue triangles represent signaling pathways. The edges represent the interactions between them.

3.9. Molecular Docking. For gaining docking affinity, LeDock software was used to dock quercetin; 7-methoxy-2methyl isoflavone; (2R)-7-hydroxy-2-(4-hydroxyphenyl) chroman-4-one; beta-sitosterol; kaempferol, and other XFZY bioactive components with AR, ESR1, PTGS2, NOS2, and other targets, respectively (Table 2). The affinity of compounds and targets is greater than $-4.25 \mathrm{kcal} / \mathrm{mol}$, which indicates that the compound had a certain affinity for the protein crystal structure. Quercetin and AR, quercetin and PTGS2, kaempferol and DPP4, kaempferol and AR, luteolin and PPARG, and luteolin and DPP4 were selected for visualization with Chimera 1.14 software and SwissDock (http://www.swissdock.ch/) (Figure 9).

\section{Discussion}

TCM has obvious advantages in treating many diseases, but it also has many problems. For example, on the one hand, the clinical efficacy evaluation is incomplete or lacks a large amount of clinical data support. To solve the problem, meta-analysis was performed. Meta-analysis is a systematic, quantitative, and comprehensive statistical method based on previous research results. It is often used to evaluate intervention randomized controlled trials in evidence-based medicine, which can objectively evaluate the clinical efficacy. Therefore, in this article, we compiled the current evidence on the total effect of XFZY on CHD in 1,844 individuals from 21 studies, and electrocardiogram efficacy of XFZY on CHD in 674 individuals from 8 studies. This evidence objectively suggested that XFZY has a sound clinical effect on CHD and there are no significant adverse reactions.

In clinical practice, blood lipids are usually used as an indicator to evaluate the curative effect. Of course, it assuredly is too limited to evaluate the effectiveness and safety of CHD in terms of total effect and electrocardiogram efficacy. Unfortunately, inclusion criteria have no other enough indicators to draw forest plot.

On the other hand, the composition of TCM is complex and the mechanism of action has not been effectively elaborated, which are the biggest bottleneck in the development and popularization of TCM. Hence, we used network pharmacology to identify major components and elucidate mechanisms of action. Network pharmacology comprehends disease as an interconnected complex biological network and cognizes the mechanisms of TCM action via network topology [33]. It is a promising approach to expound the mechanisms of TCM [34]. The results of C-T and C-T-P networks showed that quercetin; 7-methoxy-2methyl isoflavone; (2R)-7-hydroxy-2-(4-hydroxyphenyl) chroman-4-one; beta-sitosterol; kaempferol; luteolin; medicarpin; formononetin; and shinpterocarpin; 2-[(3R)-8,8dimehyl-3,4-dihydro-2H-pyrano[6,5-f]chromen-3-yl-5-

mehoxyphenol are the main ingredients at work according degree from large to small when XFZY plays curative effect and that the main targets are as follows: AR, ESR1, PTGS2, NOS2, PPARG, PIM1, DPP4, GSK3B, PRSS1, and ESR2, which mainly involves the following five signaling pathways, namely, TNF signaling pathway, pathways in cancer, calcium signaling pathway, HIF-1 signaling pathway, and Chagas disease (American trypanosomiasis).

According to the TCM prescription principles of monarch, minister, assistant, and guide, Prunus persica (L.) Batsch and Carthamus tinctorius L. are the monarch drug, Achyranthes bidentata BI, Ligusticum chuanxiong Hort, and Paeonia lactiflora Pall are the minister drug, Rehmannia glutinosa Libosch, Angelica sinensis (Oliv) Diels, Citrus aurantium L., and Platycodon grandifloras (Jacq.) A. DC are 
TABLE 2: Molecular docking of bioactive ingredients of XFZY and corresponding targets.

\begin{tabular}{|c|c|c|c|}
\hline Ingredient & Target name & PDB ID & Affinity $(\mathrm{kcal} / \mathrm{mol})$ \\
\hline Quercetin & $\mathrm{AR}$ & 2 pir & -6.90 \\
\hline Quercetin & PTGS2 & $5 \mathrm{f} 1 \mathrm{a}$ & -7.07 \\
\hline Quercetin & P1M1 & $203 p$ & -6.27 \\
\hline Quercetin & NOS2 & 4 ux6 & -6.72 \\
\hline 7-Methoxy-2-methyl isoflavone & PTGS2 & $5 \mathrm{fla}$ & -4.88 \\
\hline (2R)-7-Hydroxy-2-(4-hydroxyphenyl)chroman-4-one & P1M1 & $203 p$ & -4.59 \\
\hline Kaempferol & $\mathrm{DPP} 4$ & $5 \mathrm{t} 4 \mathrm{f}$ & -5.96 \\
\hline Kaempferol & AR & 2pir & -6.77 \\
\hline Kaempferol & ESR1 & $2 \mathrm{i} 0 \mathrm{j}$ & -6.09 \\
\hline Kaempferol & PPARG & $6 c 5 t$ & -5.97 \\
\hline Luteolin & PPARG & 2 pob & -5.75 \\
\hline Luteolin & AR & 2 pir & -6.74 \\
\hline Luteolin & DPP4 & $5 \mathrm{t} 4 \mathrm{f}$ & -6.01 \\
\hline Medicarpin & ESR1 & $2 \mathrm{i} 0 \mathrm{j}$ & -4.89 \\
\hline Formononetin & PRSS1 & 1 trn & -5.08 \\
\hline Shinpterocarpin & PPARG & $6 c 5 t$ & -4.53 \\
\hline 2-[(3R)-8,8-Dimethyl-3,4-dihydro-2H-pyrano[6,5-f]chromen-3-yl]-5-methoxyphenol & DPP4 & $5 \mathrm{y} 7 \mathrm{k}$ & -4.95 \\
\hline
\end{tabular}

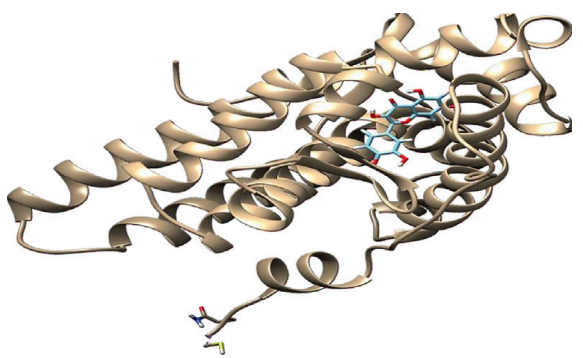

(a)

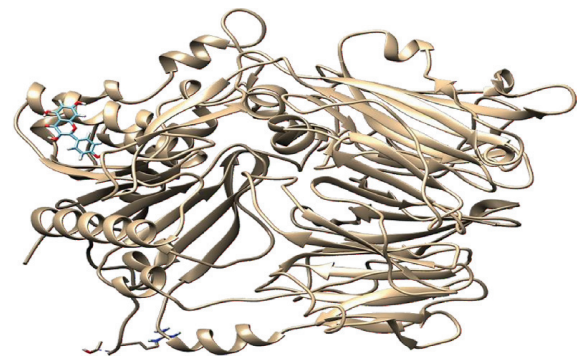

(c)

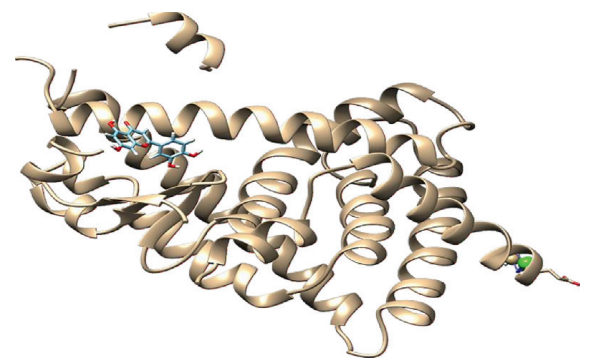

(e)

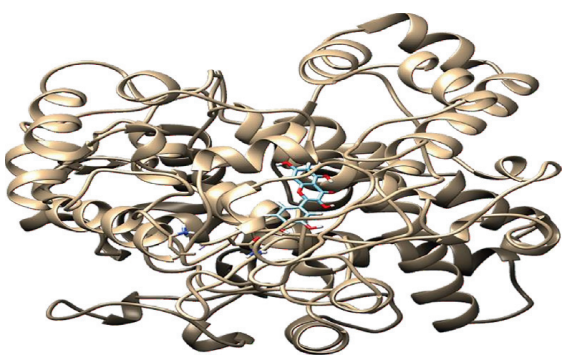

(b)

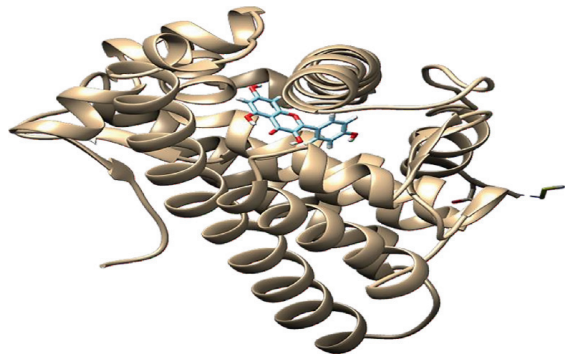

(d)

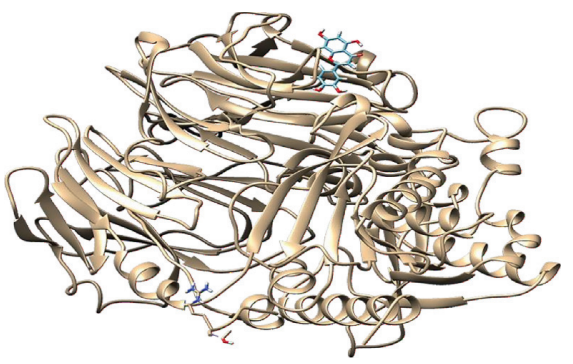

(f)

FIGURE 9: Pattern diagram of molecular docking: (a) quercetin-AR; (b) quercetin-PTGS2; (c) kaempferol-DPP4; (d) kaempferol1-AR; (e) luteolin-PPARG; (f) luteolin-DPP4.

the assistant drug, and Glycyrrhiza uralensis Fisch is the guide drug. Carthamus tinctorius L. and Prunus persica (L.) Batsch can activate blood and dissolve stasis. Paeonia lactiflora Pall, Ligusticum chuanxiong Hort, and Achyranthes bidentata BI promote stasis metabolism and excretion. Rehmannia glutinosa Libosch, Platycodon grandifloras 
(Jacq.) A. DC, Citrus aurantium L., and Angelica sinensis (Oliv) Diels clear heat and activate blood, promoting circulation of Qi and blood. Glycyrrhiza uralensis Fisch harmonizes other medicines. In the supplemental document, we find main ingredients as follows: quercetin which originates from Bupleurum chinensie DC, Glycyrrhiza uralensis Fisch, Achyranthes bidentata BI, and Carthamus tinctorius L.; 7methoxy-2-methyl isoflavone which originates from Glycyrrhiza uralensis Fisch; (2R)-7-hydroxy-2-(4-hydroxyphenyl)chroman-4-one which originates from Glycyrrhiza uralensis Fisch; beta-sitosterol which originates from Paeonia lactiflora Pall, Carthamus tinctorius L., Angelica sinensis (Oliv) Diels, Citrus aurantium L., Achyranthes bidentata BI, and Prunus persica (L.) Batsch; kaempferol which originates from Bupleurum chinensie DC, Carthamus tinctorius L., Glycyrrhiza uralensis Fisch, and Achyranthes bidentata $\mathrm{BI}$; luteolin which originates from Carthamus tinctorius L. and Platycodon grandifloras (Jacq.) A. DC; medicarpin which originates from Glycyrrhiza uralensis Fisch; formononetin which originates from Glycyrrhiza uralensis Fisch; shinpterocarpin which originates from Glycyrrhiza uralensis Fisch; 2-[(3R)-8,8-dimehyl-3,4-dihydro-2H-pyrano[6,5-f]chromen-3-yl-5-mehoxyphenol which originates from Glycyrrhiza uralensis Fisch.

Quercetin is an important flavonoid that has been normally viewed as a great antioxidant and anti-inflammatory molecule [35-37] and can prevent endothelial dysfunction and myocardial ischemia [38]. Beta-sitosterol exerted protective actions against myocardial injury [39] and is used in the treatment of hypercholesterolemia [40]. Kaempferol has antioxidant, anti-inflammatory effect [41] and protects against cardiac hypertrophy [42]. Luteolin can improve ventricular function and reduce coronary reperfusion [43] and thrombotic tendency [44]. Medicarpin and its metabolites were transported in most organs of the body [45] and can promote the publication of other ingredients. Formononetin substantially attenuates the generation of atherosclerosis [46]. 7-methoxy-2-methyl isoflavone; (2R)7-hydroxy-2-(4-hydroxyphenyl)chroman-4-one; shinpterocarpin; and 2-[(3R)-8,8-dimehyl-3,4-dihydro-2H-pyrano [6,5-f]chromen-3-yl-5-mehoxyphenol can reduce the incidence of ischemia reperfusion injuries and chronic inflammatory disease. It is well known that reducing inflammatory factor can attenuate the development of cardiac hypertrophy possibly [47]. The above ten compounds can adjust a variety of physiological activities for the treatment of coronary heart disease. The literature confirms the predicted results of network pharmacology. Generally speaking, the action of traditional Chinese medicine is consistent with the pharmacological action of its components.

The molecular docking results showed that the active components were well bound to target proteins. The affinity of quercetin and $\mathrm{AR}$ is $-6.90 \mathrm{kcal} / \mathrm{mol}$. The affinity of quercetin and PTGS2 is $-7.07 \mathrm{kcal} / \mathrm{mol}$. The affinity of kaempferol and DPP4 is $-5.96 \mathrm{kcal} / \mathrm{mol}$. The affinity of kaempferoll and AR is $-6.77 \mathrm{kcal} / \mathrm{mol}$. The affinity of luteolin and PPARG is $-5.75 \mathrm{kcal} / \mathrm{mol}$. The affinity of luteolin and DPP4 is $-6.01 \mathrm{kcal} / \mathrm{mol}$.
Androgens work by binding to androgen receptors. Androgen is a key early event in atherosclerosis [48]. ESR1 and ESR2 mediate estrogen action. Estrogen protects against atherosclerosis [49]. PTGS2 has been linked to atherosclerosis, stroke, and other CVDs [50]. NOS2 is a subtype of NOS, which can affect the composition of NO. NO bioavailability plays a vital role in the pathophysiology of cardiovascular disease [51]. Peroxisome proliferator-activated receptor gamma (PPARG) is involved in the transcription of atherosclerosis and related diseases [52]. PIM1 has cardioprotective action [53]. The loss of DPP4 activity may affect the antithrombogenic nature [54]. Manipulating GSK-3beta is a promising strategy for myocardial protection in coronary artery disease and heart failure [55].PRSS1 is mainly involved in proteolysis and digestion.

In addition to pathways in cancer and Chagas disease (American trypanosomiasis), the pathway of Xuefu Zhuyu Decoction in the treatment of CHD is also involved in the following signaling pathways: TNF signaling pathway, calcium signaling pathway, and HIF-1 signaling pathway. TNF- $\alpha$ increasing ROS levels and decreasing nitric oxide production in blood vessels leads to endothelial dysfunction. It contributes also to the development of atherosclerotic plaques [56]. Dysregulation of these $\mathrm{Ca}^{2+}$ fluxes will lead to different heart and vascular pathologies [57]. HIF plays an essential role in the complex progression of atherosclerosis [58].

According to the literature, it is not difficult to find that the targets of network pharmacology are related to the regulation of various physiological functions such as reducing inflammatory response, sex hormone regulation, NO regulation, and calcium ion regulation. Furthermore, the pathways are mainly involving calcium regulation, arteriosclerosis development, and other biological functions.

These results will provide a reliable quality evaluation standard for the treatment of CHD by Xuefu Zhuyu Decoction. The existing TCM quality evaluation system needs more effective supplement to control the quality of compound prescription general [59]. Usually, content-based quality control is a commonly adopted method at present, which may not ensure biological activity in vivo or cannot be absorbed effectually. Network pharmacology can intuitively reflect the importance of components according degree. When evaluating the quality of traditional Chinese medicinal materials, the ingredients in the C-T network with high degree value should be considered first, rather than those with high content. For XFZY treat CHD, ten compounds, quercetin; 7-methoxy-2-methyl isoflavone; (2R)-7hydroxy-2-(4-hydroxyphenyl)chroman-4-one; beta-sitosterol; kaempferol; luteolin; medicarpin; formononetin; shinpterocarpin; and 2-[(3R)-8,8-dimehyl-3,4- dihydro-2H-pyrano[6,5f]chromen-3-yl-5-mehoxyphenol, are suggested to selected as marker compounds for quality control. According to the importance of ingredients in the treatment of diseases, we can establish a quality control system more in line with the rules of TCM medication.

\section{Conclusion}

In summary, XFZY has the curative effect on coronary heart disease and its mechanism is related to 10 compounds, 10 
core targets, and 5 pathways. This study may provide a novel strategy for the understanding of TCM mechanism and make a positive contribution to the standardization of quality control. However, there may still be some potential shortcomings in this study. For example, this study lacks further experiment and more clinical sample.

\section{Data Availability}

The data used to support the findings of the study are available from the corresponding author upon request

\section{Conflicts of Interest}

The authors have declared no conflicts of interest.

\section{Authors' Contributions}

Fuguang Kui and Gang Jun Du conceived and designed the experiments. Fuguang Kui, Wenwen Gu, Fan Gao, Niu Yuji, Li Wenwen, Zhang Yaru, Guo Lijuan, Wang Junru, ZhenZhen Guo, and Shihong Cen performed the experiments. Fuguang Kui, Gang Jun Du, Zhen-Zhen Guo, and Shihong Cen analyzed the data. Zhen-Zhen Guo and Gang Jun Du contributed reagents/materials/analysis tools. Fuguang Kui, Zhen-Zhen Guo, and Gang Jun Du wrote the paper and plotted the results.

\section{Acknowledgments}

This study was supported by the Joint Foundation of $\mathrm{Na}$ tional Natural Science Foundation of China and Henan Province of China (no. U200410711). This study was supported by Youth Natural Science Foundation of Henan Province of China (no. 212300410109) and Scientific Research Foundation for Postdoctoral Science Foundation of Henan Province of China.

\section{Supplementary Materials}

Supplementary File 1: a detailed description of the methods and results of literature retrieval. Supplementary File 2: 241 compounds of XFZY, from the TCMSP database, met the principle of both oral bioavailability (OB) value $\geq 30 \%$ and drug similarity (DL) value $\geq 0.18$. The number of potential active compounds from CS (Paeonia lactiflora Pall), CX (Ligusticum chuanxiong Hort), CH (Bupleurum chinensie DC), $\mathrm{HH}$ (Carthamus tinctorius L.), DG (Angelica sinensis (Oliv) Diels), TR (Prunus persica (L.) Batsch), NX (Achyranthes bidentata BI), GC (Glycyrrhiza uralensis Fisch), JG (Platycodon grandifloras (Jacq.) A. DC), and ZK (Citrus aurantium L.) was $27,7,17,22,2,23,20,92,7$, and 5, respectively. 19 chemical moieties of SD (Rehmannia glutinosa Libosch) were collected from the Chemistry Database. Supplementary File 3 Protein targets of 241 corresponding components were obtained from TCMSP and Chemistry Database. Supplementary File 4: literature exclusion reasons. Supplementary File 5: 6785 targets of CHD were obtained from TTD, OMIM, and GeneCards. (Supplementary Materials)

\section{References}

[1] F. J. Wolters, R. A. Segufa, S. K. L. Darweesh et al., "Coronary heart disease, heart failure, and the risk of dementia: a systematic review and meta-analysis," Alzheimer's \& Dementia, vol. 14, no. 11, pp. 1493-1504, 2018.

[2] H. Li, J. Deng, L. Deng, X. Ren, and J. Xia, "Safety profile of traditional Chinese herbal injection: an analysis of a spontaneous reporting system in China," Pharmacoepidemiology and Drug Safety, vol. 28, no. 7, pp. 1002-1013, 2019.

[3] Z. Guo, M. Meng, S. Geng et al., "The optimal dose of arsenic trioxide induced opposite efficacy in autophagy between K562 cells and their initiating cells to eradicate human myelogenous leukemia," Journal of Ethnopharmacology, vol. 196, pp. 29-38, 2017.

[4] G. Zhang, G. Yang, Y. Deng et al., "Ameliorative effects of Xue-Fu-Zhu-Yu decoction, Tian-Ma-Gou-Teng-Yin and Wen-Dan decoction on myocardial fibrosis in a hypertensive rat mode," BMC Complementary and Alternative Medicine, vol. 16, p. 56, 2016.

[5] T. Tao, T. He, X. Wang, and X. Liu, "Metabolic profiling analysis of patients with coronary heart disease undergoing Xuefu Zhuyu Decoction treatment," Frontiers in Pharmacology, vol. 10, p. 985, 2019.

[6] G. Z. Yi, Y. Q. Qiu, Y. Xiao, and L. X. Yuan, “The usefulness of Xuefu Zhuyu tang for patients with angina pectoris: a metaanalysis and systematic review," Evidence-Based Complementary and Alternative Medicine, vol. 2014, Article ID 521602, , 2014.

[7] T. Yang, X. Li, Z. Lu, X. Han, and M. Zhao, "Effectiveness and safety of Xuefu Zhuyu decoction for treating coronary heart disease angina: a systematic review and meta-analysis," Medicine (Baltimore), vol. 98, no. 9, 2019.

[8] Y. Li, K. Chen, and Z. Shi, "Effect of Xuefu Zhuyu pill on blood stasis syndrome and risk factor of atherosclerosis," Zhongguo Zhong Xi Yi Jie He Za Zhi, vol. 18, no. 2, pp. 71-73, 1998.

[9] X.-Y. Lu, H. Xu, T. Zhao, and G. Li, "Study of serum metabonomics and formula-pattern correspondence in coronary heart disease patients diagnosed as phlegm or blood stasis pattern based on ultra performance liquid chromatography mass spectrometry," Chinese Journal of Integrative Medicine, vol. 24, no. 12, pp. 905-911, 2018.

[10] X. Shi, H. Zhu, Y. Zhang, M. Zhou, D. Tang, and H. Zhang, "Xuefu Zhuyu decoction protected cardiomyocytes against hypoxia/reoxygenation injury by inhibiting autophagy," BMC Complementary and Alternative Medicine, vol. 17, no. 1, pp. 1-10, 2017

[11] Y.-Y. Zhang, Z.-D. Zhao, P.-Y. Kong et al., “A comparative pharmacogenomic analysis of three classic TCM prescriptions for coronary heart disease based on molecular network modeling," ACTA Pharmacologica Sinica, vol. 41, no. 6, pp. 735-744, 2020.

[12] J. Li, "Application of Xuefu Zhuyu decoction in the treatment of angina pectoris and analysis of vascular endothelial function index," Electronic Journal of Clinical Medicine, vol. 6, no. 48 , pp. 142-143, 2019.

[13] J. Lu, "Clinical effect observation of treatment of coronary heart disease angina pectoris with Xuefu Zhuyu Decoction," Forum of Primary Medicine, vol. 22, no. 14, pp. 1964-1966, 2018.

[14] L. Jiao, "Clinical experience of treating coronary heart disease angina patients with Xuefu Zhuyu decoction," Chinese Medical Guide, vol. 13, no. 20, pp. 198-199, 2015. 
[15] D. Zhao and Yang, "Clinical observation of 50 cases of coronary heart disease angina pectoris (heart blood stasis type) treated by jiawei Xuefu Zhuyu decoction," China Sanatoria Medicine, vol. 24, no. 02, pp. 150-151, 2015.

[16] W. Huang, "Clinical observation of 36 cases of coronary heart disease angina pectoris treated by Xuefuzhuyu Decoction combined with western medicine," Hunan Journal of Traditional Chinese Medicine, vol. 34, no. 9, pp. 7-9, 2018.

[17] T. Li, "Clinical observation of Xuefu Zhuyu decoction combined with western medicine in the treatment of angina pectoris of coronary heart disease," Guangming Chinese Medicine, vol. 34, no. 13, pp. 2052-2054, 2019.

[18] Y. Fang, "Clinical observation of 60 cases of coronary atherosclerotic heart disease angina pectoris treated by Xuefu Zhuyu decoction combined with western medicine," Hebei Chinese Medicine, vol. 31, no. 5, pp. 711-712, 2009.

[19] P. Ying, "Clinical observation of Xuefu Zhuyu decoction combined with western medicine for angina pectoris," New Chinese Medicine, vol. 50, no. 8, pp. 40-42, 2018.

[20] B. Wang, "Treatment of 58 cases of coronary heart disease with Xuefu Zhuyu decoction," Chinese Traditional Chinese Medicine Modern Distance Education, vol. 8, no. 15, p. 34, 2010.

[21] J. Fang, "Clinical observation of 30 cases of angina pectoris with coronary heart disease treated by Xuefu Zhuyu decoction," Guangzhou Western Medicine, vol. 30, no. 9, pp. 1352-1353, 2008.

[22] Z. Lin, "Effect analysis of Xuefu Zhuyu decoction on angina pectoris of coronary heart disease," Clinical Research of Traditional Chinese Medicine, vol. 7, no. 26, pp. 71-72, 2015.

[23] Y. Song, "Clinical observation on the treatment of angina pectoris by Xuefu Zhuyu decoction," Journal of Nantong University (Medical Edition), vol. 33, no. 5, pp. 416-417, 2013.

[24] X. Lv, "Therapeutic effect of Xuefu Zhuyu decoction on angina pectoris of coronary heart disease," Heilongjiang Science, vol. 10, no. 14, pp. 128-129, 2019.

[25] G. Li, "Effect of Xuefu Zhuyu decoction on angina pectoris of coronary heart disease," Henan Chinese Medicine, vol. 29, no. 12, pp. 1226-1227, 2009.

[26] S. Yang, "Therapeutic effect of Xuefu Zhuyu decoction on angina pectoris of coronary heart disease," Chinese Medicine Pharmacology and Clinical Practice, vol. 31, no. 6, pp. 144-146, 2015.

[27] G. Zhang, "Therapeutic effect of Xuefu Zhuyu decoction on angina pectoris of coronary heart disease," Wisdom and Health, vol. 4, no. 27, pp. 110-111, 2018.

[28] L. Liang and J. Li, "Therapeutic effect of Xuefu Zhuyu decoction combined with western medicine in treating coronary heart disease angina pectoris with blood stasis syndrome," World Latest Medical Information Digest, vol. 15, no. 82, pp. 21-22, 2015.

[29] X. Cai, "Clinical effect analysis of Xuefu Zhuyu decoction combined with western medicine in patients with coronary heart disease angina pectoris with blood stasis syndrome," Chinese Journal of Modern Medicine, vol. 11, no. 5, pp. 182-183, 2017.

[30] F. Zhou, "Effect of Xuefu Zhuyu decoction on angina pectoris of coronary heart disease with Qi deficiency and blood stasis," Chinese Medical Forum, vol. 34, no. 3, pp. 39-40, 2019.

[31] M. Zhang and P. Wang, "Therapeutic effect of Xuefu Zhuyu decoction combined with western medicine in treating coronary heart disease angina pectoris with blood stasis syndrome," Hubei Journal of Traditional Chinese Medicine, vol. 32, no. 4, pp. 16-17, 2010.
[32] L. Chen, "Clinical observation on the treatment of angina pectoris with coronary heart disease by Xuefu Zhuyu decoction," Shaanxi Traditional Chinese Medicine, vol. 36, no. 7, pp. 812-813, 2015.

[33] W. Y. Lee, C. Y. Lee, Y. S. Kim, and C. E. Kim, "The methodological trends of traditional herbal medicine employing network pharmacology," Biomolecules, vol. 9, no. 8, 2019.

[34] E. L. Berg, "Systems biology in drug discovery and development," Drug Discovery Today, vol. 19, no. 2, pp. 113-125, 2014.

[35] M. R. De Oliveira, S. M. Nabavi, N. Braidy, W. N. Setzer, T. Ahmed, and S. F. Nabavi, "Quercetin and the mitochondria: a mechanistic view," Biotechnology Advances, vol. 34, no. 5, pp. 532-549, 2016.

[36] F. Xue, X. Nie, J. Shi et al., "Quercetin inhibits lps-induced inflammation and ox-ldl-induced lipid deposition," Frontiers in Pharmacology, vol. 8, p. 40, 2017.

[37] Y. Zhang and Y. Zhang, "Pterostilbene, a novel natural plant conduct, inhibits high fat-induced atherosclerosis inflammation via NF- $\kappa \mathrm{B}$ signaling pathway in Toll-like receptor 5 (TLR5) deficient mice," Biomedicine \& Pharmacotherapy, vol. 81, pp. 345-355, 2016.

[38] F. Perez-Vizcaino and J. Duarte, "Flavonols and cardiovascular disease," Molecular Aspects of Medicine, vol. 31, no. 6, pp. $478-494,2010$.

[39] F. Lin, L. Xu, M. Huang et al., " $\beta$-Sitosterol protects against myocardial ischemia/reperfusion injury via targeting PPAR $\gamma /$ NF- $\kappa \mathrm{B}$ signalling," Evidence Based Complementary Alternative Medicine, vol. 2020, Article ID 2679409, 2020.

[40] S. Ramalingam, M. Packirisamy, M. Karuppiah et al., "Effect of $\beta$-sitosterol on glucose homeostasis by sensitization of insulin resistance via enhanced protein expression of PPR $\gamma$ and glucose transporter 4 in high fat diet and streptozotocininduced diabetic rats," Cytotechnology, vol. 72, no. 3, pp. 357-366, 2020.

[41] I. Crespo, M. V. García-Mediavilla, B. Gutiérrez, S. SánchezCampos, M. J. Tuñón, and J. González-Gallego, "A comparison of the effects of kaempferol and quercetin on cytokine-induced pro-inflammatory status of cultured human endothelial cells," British Journal of Nutrition, vol. 100, no. 5, pp. 968-976, 2008.

[42] H. Feng, J. Cao, G. Zhang, and Y. Wang, "Kaempferol attenuates cardiac hypertrophy via regulation of ask1/mapk signaling pathway and oxidative stress," Planta Medica, vol. 83, no. 10, pp. 837-845, 2017.

[43] J.-T. Yang, L.-B. Qian, F.-J. Zhang et al., "Cardioprotective effects of luteolin on ischemia/reperfusion injury in diabetic rats are modulated by eNOS and the mitochondrial permeability transition pathway," Journal of Cardiovascular Pharmacology, vol. 65, no. 4, pp. 349-356, 2015.

[44] M. G. L. Hertog, E. J. M. Feskens, D. Kromhout et al., "Dietary antioxidant flavonoids and risk of coronary heart disease: the zutphen elderly study," The Lancet, vol. 342, no. 8878, pp. 1007-1011, 1993.

[45] H. Y. Wang, T. Li, R. Ji et al., "Metabolites of medicarpin and their distributions in rats," Molecules, vol. 24, no. 10, 2019.

[46] C. Ma, R. Xia, S. Yang et al., "Formononetin attenuates atherosclerosis via regulating interaction between KLF4 and SRA in apoE-/- mice," Theranostics, vol. 10, no. 3, pp. 1090-1106, 2020.

[47] Y. Li, J. Wang, L. Sun, and S. Zhu, "LncRNA myocardial infarction-associated transcript (MIAT) contributed to 
cardiac hypertrophy by regulating TLR4 via miR-93," European Journal of Pharmacology, vol. 818, pp. 508-517, 2018.

[48] Y. Ikeda, K.-I. Aihara, S. Yoshida et al., "Androgen-androgen receptor system protects against angiotensin II-induced vascular remodeling," Endocrinology, vol. 150, no. 6, pp. 2857-2864, 2009.

[49] A. D. P. Mansur, C. C. M. Nogueira, C. M. C. Strunz, J. M. Aldrighi, and J. A. F. Ramires, "Genetic polymorphisms of estrogen receptors in patients with premature coronary artery disease," Archives of Medical Research, vol. 36, no. 5, pp. 511-517, 2005.

[50] E. Berinstein and A. Levy, "Recent developments and future directions for the use of pharmacogenomics in cardiovascular disease treatments," Expert Opinion on Drug Metabolism \& Toxicology, vol. 13, no. 9, pp. 973-983, 2017.

[51] T. Fernandes, C. V. Gomes-Gatto, N. P. Pereira, Y. R. Alayafi, V. J. Das Neves, and E. M. Oliveira, "NO signaling in the cardiovascular system and exercise," Advances in Experimental Medicine and Biology, vol. 1000, pp. 211-245, 2017.

[52] W. M. We, X. Y. Wu, S. T. Li, and Q. Shen, "PPARG gene C161T CT/TT associated with lower blood lipid levels and ischemic stroke from large-artery atherosclerosis in a Han population in Guangdong," Neurological Research, vol. 38, no. 7, pp. 620-624, 2016.

[53] G. A. Borillo, M. Mason, P. Quijada et al., "Pim-1 kinase protects mitochondrial integrity in cardiomyocytes," Circulation Research, vol. 106, no. 7, pp. 1265-1274, 2010.

[54] P. A. J. Krijnen, N. E. Hahn, I. Kholová et al., "Loss of DPP4 activity is related to a prothrombogenic status of endothelial cells: implications for the coronary microvasculature of myocardial infarction patients," Basic Research in Cardiology, vol. 107, no. 1, 2012.

[55] T. Miura and T. Miki, "GSK-3 $\beta$, a therapeutic target for cardiomyocyte protection," Circulation Journal, vol. 73, no. 7, pp. 1184-1192, 2009.

[56] F. Rolski and P. Błyszczuk, "Complexity of TNF- $\alpha$ signaling in heart disease," Journal of Clinical Medicine, vol. 9, no. 10, p. $3267,2020$.

[57] A. Ghigo, M. Laffargue, M. Li, and E. Hirsch, "PI3K and calcium signaling in cardiovascular disease," Circulation Research, vol. 121, no. 3, pp. 282-292, 2017.

[58] T. Jain, E. A. Nikolopoulou, Q. Xu, and A. Qu, "Hypoxia inducible factor as a therapeutic target for atherosclerosis," Pharmacology \& Therapeutics, vol. 183, no. xxxx, pp. 22-33, 2018.

[59] X. Wu, H. Zhang, S. Fan et al., "Quality markers based on biological activity: a new strategy for the quality control of traditional Chinese medicine," Phytomedicine, vol. 44, pp. 103-108, 2018. 